\title{
Optimal evaluation of a Toader-type mean by power mean
}

\author{
Ying-Qing Song ${ }^{1}$, Tie-Hong Zhao ${ }^{2}$, Yu-Ming Chu ${ }^{1 *}$ and Xiao-Hui Zhang'
}

"Correspondence:
chuyuming2005@126.com
'School of Mathematics and
Computation Science, Hunan City
University, Yiyang, 413000, China
Full list of author information is
available at the end of the article

\begin{abstract}
In this paper, we present the best possible parameters $p, q \in \mathbb{R}$ such that the double inequality $M_{p}(a, b)<T[A(a, b), Q(a, b)]<M_{q}(a, b)$ holds for all $a, b>0$ with $a \neq b$, and we get sharp bounds for the complete elliptic integral $\mathcal{E}(t)=\int_{0}^{\pi / 2}\left(1-t^{2} \sin ^{2} \theta\right)^{1 / 2} d \theta$ of the second kind on the interval $(0, \sqrt{2} / 2)$, where

$T(a, b)=\frac{2}{\pi} \int_{0}^{\pi / 2} \sqrt{a^{2} \cos ^{2} \theta+b^{2} \sin ^{2} \theta} d \theta, A(a, b)=(a+b) / 2, Q(a, b)=\sqrt{\left(a^{2}+b^{2}\right) / 2}$, $M_{r}(a, b)=\left[\left(a^{r}+b^{r}\right) / 2\right]^{1 / r}(r \neq 0)$, and $M_{0}(a, b)=\sqrt{a b}$ are the Toader, arithmetic, quadratic, and $r$ th power means of $a$ and $b$, respectively.
\end{abstract}

MSC: 33E05; 33C05; 26E60

Keywords: arithmetic mean; Toader mean; quadratic mean

\section{Introduction}

For $r \in \mathbb{R}$ and $a, b>0$, the Toader mean $T(a, b)$ (see [1]) and $r$ th power mean $M_{r}(a, b)$ are defined by

$$
T(a, b)=\frac{2}{\pi} \int_{0}^{\pi / 2} \sqrt{a^{2} \cos ^{2} \theta+b^{2} \sin ^{2} \theta} d \theta
$$

and

$$
M_{r}(a, b)= \begin{cases}\left(\frac{a^{r}+b^{r}}{2}\right)^{1 / r}, & r \neq 0, \\ \sqrt{a b}, & r=0,\end{cases}
$$

respectively.

It is well known that $M_{r}(a, b)$ is continuous and strictly increasing with respect to $r \in \mathbb{R}$ for fixed $a, b>0$ with $a \neq b$. Many classical bivariate means are a special case of the power mean, for example, $H(a, b)=2 a b /(a+b)=M_{-1}(a, b)$ is the harmonic mean, $G(a, b)=\sqrt{a b}=$ $M_{0}(a, b)$ is the geometric mean,

$$
A(a, b)=(a+b) / 2=M_{1}(a, b)
$$

is the arithmetic mean, and

$$
Q(a, b)=\sqrt{\left(a^{2}+b^{2}\right) / 2}=M_{2}(a, b)
$$

(c) 2015 Song et al. This article is distributed under the terms of the Creative Commons Attribution 4.0 International License (http://creativecommons.org/licenses/by/4.0/), which permits unrestricted use, distribution, and reproduction in any medium, provided you give appropriate credit to the original author(s) and the source, provide a link to the Creative Commons license, and indicate if changes were made. 
is the quadratic mean. The main properties of the power mean are given in [2]. The Toader mean $T(a, b)$ has been well known in the mathematical literature for many years, it satisfies

$$
T(a, b)=R_{E}\left(a^{2}, b^{2}\right)
$$

where

$$
R_{E}(a, b)=\frac{1}{\pi} \int_{0}^{\infty} \frac{[a(t+b)+b(t+a)] t}{(t+a)^{3 / 2}(t+b)^{3 / 2}} d t
$$

stands for the symmetric complete elliptic integral of the second kind (see [3-5]), therefore it cannot be expressed in terms of the elementary transcendental functions.

Let $r \in(0,1), \mathcal{K}(r)=\int_{0}^{\pi / 2}\left(1-r^{2} \sin ^{2} \theta\right)^{-1 / 2} d \theta$, and $\mathcal{E}(r)=\int_{0}^{\pi / 2}\left(1-r^{2} \sin ^{2} \theta\right)^{1 / 2} d \theta$ be, respectively, the complete elliptic integrals of the first and second kind. Then $\mathcal{K}\left(0^{+}\right)=$ $\mathcal{E}\left(0^{+}\right)=\pi / 2$, the Toader mean $T(a, b)$ given in (1.1) can be expressed as

$$
T(a, b)= \begin{cases}\frac{2 a}{\pi} \mathcal{E}\left(\sqrt{1-\left(\frac{b}{a}\right)^{2}}\right), & a>b, \\ \frac{2 b}{\pi} \mathcal{E}\left(\sqrt{1-\left(\frac{a}{b}\right)^{2}}\right), & a<b,\end{cases}
$$

and $\mathcal{K}(r)$ and $\mathcal{E}(r)$ satisfy the derivatives formulas (see [6], Appendix E, p. 474-475)

$$
\frac{d \mathcal{K}(r)}{d r}=\frac{\mathcal{E}(r)-\left(1-r^{2}\right) \mathcal{K}(r)}{r\left(1-r^{2}\right)}, \quad \frac{d \mathcal{E}(r)}{d r}=\frac{\mathcal{E}(r)-\mathcal{K}(r)}{r}, \quad \frac{d(\mathcal{K}(r)-\mathcal{E}(r))}{d r}=\frac{r \mathcal{E}(r)}{1-r^{2}}
$$

Numerical computations show that

$$
\begin{aligned}
& \mathcal{E}\left(\frac{\sqrt{2}}{2}\right)=1.3506 \ldots, \quad \mathcal{K}\left(\frac{3}{5}\right)=1.7507 \ldots, \quad \mathcal{E}\left(\frac{3}{5}\right)=1.4180 \ldots, \\
& \mathcal{K}\left(\frac{17}{25}\right)=1.8234 \ldots, \quad \mathcal{E}\left(\frac{17}{25}\right)=1.3693 \ldots
\end{aligned}
$$

Recently, the power mean $M_{r}(a, b)$ and Toader mean $T(a, b)$ have been the subject of intensive research. In particular, many remarkable inequalities for both means can be found in the literature [7-18].

Vuorinen [19] conjectured that the inequality

$$
M_{3 / 2}(a, b)<T(a, b)
$$

holds for all $a, b>0$ with $a \neq b$. This conjecture was proved by Qiu and Shen [20], and Barnard et al. [21], respectively.

Alzer and Qiu [22] presented a best possible upper power mean bound for the Toader mean as follows:

$$
T(a, b)<M_{\log 2 /(\log \pi-\log 2)}(a, b)
$$

for all $a, b>0$ with $a \neq b$. 
Neuman [3], and Kazi and Neuman [4] proved that the inequalities

$$
\begin{aligned}
& \frac{(a+b) \sqrt{a b}-a b}{A G M(a, b)}<T(a, b)<\frac{4(a+b) \sqrt{a b}+(a-b)^{2}}{8 A G M(a, b)}, \\
& T(a, b)<\frac{1}{4}\left(\sqrt{(2+\sqrt{2}) a^{2}+(2-\sqrt{2}) b^{2}}+\sqrt{(2+\sqrt{2}) b^{2}+(2-\sqrt{2}) a^{2}}\right)
\end{aligned}
$$

hold for all $a, b>0$ with $a \neq b$, where $\operatorname{AGM}(a, b)$ is the arithmetic-geometric mean of $a$ and $b$.

Let $\lambda, \mu, \alpha, \beta \in(1 / 2,1)$. Then Chu et al. [23], and Hua and Qi [24] proved that the double inequalities

$$
\begin{aligned}
& C[\lambda a+(1-\lambda) b, \lambda b+(1-\lambda) a]<T(a, b)<C[\mu a+(1-\mu) b, \mu b+(1-\mu) a], \\
& \bar{C}[\alpha a+(1-\alpha) b, \alpha b+(1-\alpha) a]<T(a, b)<\bar{C}[\beta a+(1-\beta) b, \beta b+(1-\beta) a]
\end{aligned}
$$

hold for all $a, b>0$ with $a \neq b$ if and only if $\lambda \leq 3 / 4, \mu \geq 1 / 2+\sqrt{\pi(4-\pi)} /(2 \pi), \alpha \leq 1 / 2+$ $\sqrt{3} / 4$, and $\beta \geq 1 / 2+\sqrt{12 / \pi-3} / 2$, where $C(a, b)=\left(a^{2}+b^{2}\right) /(a+b)$ and $\bar{C}(a, b)=2\left(a^{2}+\right.$ $\left.a b+b^{2}\right) /[3(a+b)]$ are, respectively, the contraharmonic and centroidal means of $a$ and $b$.

In [25-29], the authors proved that the double inequalities

$$
\begin{aligned}
& \alpha_{1} Q(a, b)+\left(1-\alpha_{1}\right) A(a, b)<T(a, b)<\beta_{1} Q(a, b)+\left(1-\beta_{1}\right) A(a, b), \\
& Q^{\alpha_{2}}(a, b) A^{\left(1-\alpha_{2}\right)}(a, b)<T(a, b)<Q^{\beta_{2}}(a, b) A^{\left(1-\beta_{2}\right)}(a, b), \\
& \alpha_{3} C(a, b)+\left(1-\alpha_{3}\right) A(a, b)<T(a, b)<\beta_{3} C(a, b)+\left(1-\beta_{3}\right) A(a, b), \\
& \frac{\alpha_{4}}{A(a, b)}+\frac{1-\alpha_{4}}{C(a, b)}<\frac{1}{T(a, b)}<\frac{\beta_{4}}{A(a, b)}+\frac{1-\beta_{4}}{C(a, b)}, \\
& \alpha_{5} C(a, b)+\left(1-\alpha_{5}\right) H(a, b)<T(a, b)<\beta_{5} C(a, b)+\left(1-\beta_{5}\right) H(a, b), \\
& \alpha_{6}[C(a, b)-H(a, b)]+A(a, b)<T(a, b)<\beta_{6}[C(a, b)-H(a, b)]+A(a, b), \\
& \alpha_{7} \bar{C}(a, b)+\left(1-\alpha_{7}\right) A(a, b)<T(a, b)<\beta_{7} \bar{C}(a, b)+\left(1-\beta_{7}\right) A(a, b), \\
& \frac{\alpha_{8}}{A(a, b)}+\frac{1-\alpha_{8}}{\bar{C}(a, b)}<\frac{1}{T(a, b)}<\frac{\beta_{8}}{A(a, b)}+\frac{1-\beta_{8}}{\bar{C}(a, b)}, \\
& \alpha_{9} Q(a, b)+\left(1-\alpha_{9}\right) H(a, b)<T(a, b)<\beta_{9} Q(a, b)+\left(1-\beta_{9}\right) H(a, b), \\
& \frac{\alpha_{10}}{H(a, b)}+\frac{1-\alpha_{10}}{Q(a, b)}<\frac{1}{T(a, b)}<\frac{\beta_{10}}{H(a, b)}+\frac{1-\beta_{10}}{Q(a, b)}
\end{aligned}
$$

hold for all $a, b>0$ with $a \neq b$ if and only if $\alpha_{1} \leq 1 / 2, \beta_{1} \geq(4-\pi) /[(\sqrt{2}-1) \pi], \alpha_{2} \leq 1 / 2$, $\beta_{2} \geq 4-2 \log \pi / \log 2, \alpha_{3} \leq 1 / 4, \beta_{3} \geq 4 / \pi-1, \alpha_{4} \leq \pi / 2-1, \beta_{4} \geq 3 / 4, \alpha_{5} \leq 5 / 8, \beta_{5} \geq 2 / \pi$, $\alpha_{6} \leq 1 / 8, \beta_{6} \geq 2 / \pi-1 / 2, \alpha_{7} \leq 3 / 4, \beta_{7} \geq 12 / \pi-3, \alpha_{8} \leq \pi-3, \beta_{8} \geq 1 / 4, \alpha_{9} \leq 5 / 6, \beta_{9} \geq$ $2 \sqrt{2} / \pi, \alpha_{10} \leq 0$, and $\beta_{10} \geq 1 / 6$.

The main purpose of this paper is to present the best possible parameters $p, q \in \mathbb{R}$ such that the double inequality

$$
M_{p}(a, b)<T[A(a, b), Q(a, b)]<M_{q}(a, b)
$$

holds for all $a, b>0$ with $a \neq b$. 


\section{Lemmas}

In order to prove our main results, we need several lemmas which we present in this section.

Lemma 2.1 (See [30], Theorem 1.1) The inequality $\mathcal{E}\left[M_{p}(x, y)\right]>M_{q}[\mathcal{E}(x), \mathcal{E}(y)]$ holds for all $x, y \in(0,1)$ if and only if

$$
p \leq C(q):=\inf _{r \in(0,1)}\left\{\frac{r^{2} \mathcal{E}(r)}{\left(1-r^{2}\right)[\mathcal{K}(r)-\mathcal{E}(r)]}+\frac{(1-q)[\mathcal{K}(r)-\mathcal{E}(r)]}{\mathcal{E}(r)}\right\}
$$

where $q \rightarrow C(q)$ is a continuous function which satisfies $C(q)=2$ for all $q \leq 5 / 2$ and $C(q)<$ 2 for all $q>5 / 2$.

Lemma 2.2 The double inequality

$$
\frac{\left(1-t^{2}\right)^{5 / 8}+1}{\left(1-t^{2}\right)^{1 / 8}+1}<1-\frac{t^{2}}{4}<\left[\frac{\left(\sqrt{1-t^{2}}+t\right)^{3 / 2}+\left(\sqrt{1-t^{2}}-t\right)^{3 / 2}}{2}\right]^{2 / 3}
$$

holds for all $t \in(0, \sqrt{2} / 2)$.

Proof Let $u=\left(1-t^{2}\right)^{1 / 8}$. Then $u \in(1 / \sqrt[8]{2}, 1), t^{2}=1-u^{8}$, and the first inequality of (2.1) is equivalent to

$$
\frac{u^{5}+1}{u+1}<\frac{u^{8}+3}{4}
$$

for all $u \in(1 / \sqrt[8]{2}, 1)$.

We clearly see that (2.2) follows from

$$
(u+1)\left(u^{8}+3\right)-4\left(u^{5}+1\right)=(u+1)\left(u^{2}+1\right)(1-u)^{2}\left[(2 u-1)+2 u^{2}+2 u^{3}+u^{4}\right]>0
$$

for all $u \in(1 / \sqrt[8]{2}, 1)$.

For the second inequality of (2.1), let $v=\sqrt{1-t^{2}} \in(\sqrt{2} / 2,1)$, then it suffices to prove that

$$
\begin{aligned}
\rho(v) & :=\frac{\left[\left(v+\sqrt{1-v^{2}}\right)^{3 / 2}+\left(v-\sqrt{1-v^{2}}\right)^{3 / 2}\right]^{2}}{4}-\frac{\left(v^{2}+3\right)^{3}}{64} \\
& =\frac{1}{2}\left[3 v-2 v^{3}+\left(2 v^{2}-1\right)^{3 / 2}-\frac{\left(v^{2}+3\right)^{3}}{32}\right]>0
\end{aligned}
$$

for all $v \in(\sqrt{2} / 2,1)$.

We claim that

$$
\left(2 v^{2}-1\right)^{3 / 2}>2-6 v+3 v^{2}+2 v^{3}
$$

for all $v \in(\sqrt{2} / 2,1)$. 
Indeed, if $v \in(\sqrt{2} / 2,(\sqrt{6}-1) / 2]$, then we clearly see that the function $2-6 v+3 v^{2}+2 v^{3}$ is strictly increasing on $(\sqrt{2} / 2,(\sqrt{6}-1) / 2]$, and (2.4) follows from

$$
\begin{aligned}
2-6 v+3 v^{2}+2 v^{3} & \leq 2-6 \times \frac{\sqrt{6}-1}{2}+3 \times\left(\frac{\sqrt{6}-1}{2}\right)^{2}+2 \times\left(\frac{\sqrt{6}-1}{2}\right)^{3} \\
& =\frac{22-9 \sqrt{6}}{4}<0 .
\end{aligned}
$$

If $v \in((\sqrt{6}-1) / 2,1)$, then (2.4) follows easily from

$$
\begin{aligned}
\left(2 v^{2}-1\right)^{3}-\left(2-6 v+3 v^{2}+2 v^{3}\right)^{2} & =\left(1-v^{4}\right)\left(-5+4 v+4 v^{2}\right) \\
& >\left(1-v^{4}\right)\left[-5+4 \times \frac{\sqrt{6}-1}{2}+4\left(\frac{\sqrt{6}-1}{2}\right)^{2}\right]=0 .
\end{aligned}
$$

Therefore, inequality (2.3) follows from (2.4) and

$$
\begin{aligned}
3 v-2 v^{3}+\left(2 v^{2}-1\right)^{3 / 2}-\frac{\left(v^{2}+3\right)^{3}}{32} & >3 v-2 v^{3}+\left(2-6 v+3 v^{2}+2 v^{3}\right)-\frac{\left(v^{2}+3\right)^{3}}{32} \\
& =\frac{\left(1-v^{3}\right)\left(37+15 v+3 v^{2}+v^{3}\right)}{32}>0
\end{aligned}
$$

for all $v \in(\sqrt{2} / 2,1)$.

Lemma 2.3 The inequality

$$
\mathcal{E}(t)>\frac{\pi}{2}\left(1-\frac{5 t^{2}}{18}\right)
$$

holds for all $t \in(0,3 / 5)$.

Proof Let

$$
f(t)=\mathcal{E}(t)-\frac{\pi}{2}\left(1-\frac{5 t^{2}}{18}\right) .
$$

Then simple computations lead to

$$
\begin{aligned}
& f\left(0^{+}\right)=0, \quad f\left(\frac{3}{5}\right)=0.00436 \ldots>0, \\
& f^{\prime}(t)=t f_{1}(t),
\end{aligned}
$$

where

$$
\begin{aligned}
& f_{1}(t)=\frac{\mathcal{E}(t)-\mathcal{K}(t)}{t^{2}}+\frac{5 \pi}{18} \\
& f_{1}\left(0^{+}\right)=\frac{\pi}{36}>0, \quad f_{1}\left(\frac{3}{5}\right)=-0.0514 \ldots<0 \\
& f_{1}^{\prime}(t)=\frac{f_{2}(t)}{t^{3}\left(1-t^{2}\right)}
\end{aligned}
$$


where

$$
\begin{aligned}
& f_{2}(t)=2\left(1-t^{2}\right) \mathcal{K}(t)-\left(2-t^{2}\right) \mathcal{E}(t), \\
& f_{2}\left(0^{+}\right)=0 \\
& f_{2}^{\prime}(t)=-3 t[\mathcal{K}(t)-\mathcal{E}(t)]<0
\end{aligned}
$$

for $t \in(0,3 / 5)$.

From (2.9)-(2.11) we clearly see that $f_{1}(t)$ is strictly decreasing on $(0,3 / 5)$. Then $(2.7)$ and (2.8) lead to the conclusion that there exists $t_{0} \in(0,3 / 5)$ such that $f(t)$ is strictly increasing on $\left(0, t_{0}\right]$ and strictly decreasing on $\left[t_{0}, 3 / 5\right)$.

Therefore, Lemma 2.3 follows easily from (2.5) and (2.6) together with the piecewise monotonicity of $f(t)$.

Lemma 2.4 The inequality

$$
\left(\frac{18+13 t^{2}}{18 \sqrt{1+t^{2}}}\right)^{7 / 5}>1+\frac{14 t^{2}}{45}
$$

holds for all $t \in(0,3 / 4)$.

Proof It suffices to prove that the inequalities

$$
\frac{18+13 t^{2}}{18 \sqrt{1+t^{2}}}>1+\frac{2 t^{2}}{9}
$$

and

$$
\left(1+\frac{2 t^{2}}{9}\right)^{7 / 5}>1+\frac{14 t^{2}}{45}
$$

hold for all $t \in(0,3 / 4)$.

Indeed, inequalities (2.12) and (2.13) follow easily from the identities

$$
\left(18+13 t^{2}\right)^{2}-4\left(1+t^{2}\right)\left(9+2 t^{2}\right)^{2}=t^{4}(3-4 t)(3+4 t)
$$

and

$$
\begin{aligned}
& \left(1+\frac{2 t^{2}}{9}\right)^{7}-\left(1+\frac{14 t^{2}}{45}\right)^{5} \\
& =4 t^{2}\left(\frac{7}{405}+\frac{14 t^{2}}{675}+\frac{2,632 t^{4}}{273,375}+\frac{390,544 t^{6}}{184,528,125}+\frac{112 t^{8}}{531,441}+\frac{32 t^{10}}{4,782,969}\right) .
\end{aligned}
$$

Lemma 2.5 Let $\lambda=2 \log 2 /[2 \log \pi-\log 2-2 \log \mathcal{E}(\sqrt{2} / 2)]=1.3930 \ldots$ and

$$
g(t)=\frac{2}{\pi} \sqrt{1+t^{2}} \mathcal{E}\left(\frac{t}{\sqrt{1+t^{2}}}\right)-\left[\frac{(1+t)^{\lambda}+(1-t)^{\lambda}}{2}\right]^{1 / \lambda} .
$$

Then $g(t)>0$ for all $t \in(0,3 / 4)$. 
Proof It follows from $t / \sqrt{1+t^{2}} \in(0,3 / 5), \lambda<7 / 5$, Lemma 2.3, Lemma 2.4 and the monotonicity of $M_{r}(1+t, 1-t)$ with respect to $r \in \mathbb{R}$ that

$$
\begin{aligned}
g(t) & >\frac{2}{\pi} \sqrt{1+t^{2}} \times \frac{\pi}{2}\left[1-\frac{5 t^{2}}{18\left(1+t^{2}\right)}\right]-\left[\frac{(1+t)^{7 / 5}+(1-t)^{7 / 5}}{2}\right]^{5 / 7} \\
& =\frac{18+13 t^{2}}{18 \sqrt{1+t^{2}}}-\left[\frac{(1+t)^{7 / 5}+(1-t)^{7 / 5}}{2}\right]^{5 / 7} \\
& >\left(1+\frac{14 t^{2}}{45}\right)^{5 / 7}-\left[\frac{(1+t)^{7 / 5}+(1-t)^{7 / 5}}{2}\right]^{5 / 7}
\end{aligned}
$$

for $t \in(0,3 / 4)$. Let

$$
g_{1}(t)=2\left(1+\frac{14 t^{2}}{45}\right)-\left[(1+t)^{7 / 5}+(1-t)^{7 / 5}\right]
$$

Then simple computations lead to

$$
\begin{aligned}
& g_{1}(0)=0, \quad g_{1}\left(\frac{3}{4}\right)=0.0173 \ldots>0, \\
& g_{1}^{\prime}(t)=\frac{7}{45}\left[8 t-9(1+t)^{2 / 5}+9(1-t)^{2 / 5}\right], \\
& g_{1}^{\prime}(0)=0, \quad g_{1}^{\prime}\left(\frac{3}{4}\right)=-0.0138 \ldots<0, \\
& g_{1}^{\prime \prime}(t)=\frac{14}{225}\left[20-\frac{9}{(1+t)^{3 / 5}}-\frac{9}{(1-t)^{3 / 5}}\right], \\
& g_{1}^{\prime \prime}(0)=\frac{28}{225}>0, \quad g_{1}^{\prime \prime}\left(\frac{3}{4}\right)=-0.4423 \ldots<0, \\
& g_{1}^{\prime \prime \prime}(t)=\frac{42}{125}\left[\frac{1}{(1+t)^{8 / 5}}-\frac{1}{(1-t)^{8 / 5}}\right]<0
\end{aligned}
$$

for $t \in(0,3 / 4)$.

From (2.18) and (2.19) we know that there exists $t_{1} \in(0,3 / 4)$ such that $g_{1}^{\prime}(t)$ is strictly increasing on $\left(0, t_{1}\right]$ and strictly decreasing on $\left[t_{1}, 3 / 4\right)$. Then (2.17) leads to the conclusion that there exists $t_{2} \in(0,3 / 4)$ such that $g_{1}(t)$ is strictly increasing on $\left(0, t_{2}\right]$ and strictly decreasing on $\left[t_{2}, 3 / 4\right)$.

Therefore, Lemma 2.5 follows from (2.14)-(2.16) and the piecewise monotonicity of $g_{1}(t)$.

Lemma 2.6 Let $\lambda=2 \log 2 /[2 \log \pi-\log 2-2 \log \mathcal{E}(\sqrt{2} / 2)]=1.3930 \ldots$. Then the function $t^{-1} \mathcal{E}^{\lambda-1}(t)[\mathcal{E}(t)-\mathcal{K}(t)]$ is strictly decreasing on $(0,1)$.

Proof From Lemma 2.1 we clearly see that the inequality

$$
\mathcal{E}\left(M_{\lambda}(x, y)\right)>M_{\lambda}(\mathcal{E}(x), \mathcal{E}(y))=\left(\frac{\mathcal{E}^{\lambda}(x)+\mathcal{E}^{\lambda}(y)}{2}\right)^{1 / \lambda}
$$

holds for all $x, y \in(0,1)$ with $x \neq y$. 
It follows from the monotonicity of the function $\mathcal{E}(t)$ and the power mean $M_{p}(x, y)$ with respect to $p \in \mathbb{R}$ together with $\lambda>1$ that

$$
\mathcal{E}\left(\frac{x+y}{2}\right)=\mathcal{E}\left(M_{1}(x, y)\right)>\mathcal{E}\left(M_{\lambda}(x, y)\right)
$$

for all $x, y \in(0,1)$ with $x \neq y$.

Inequalities (2.20) and (2.21) lead to

$$
\mathcal{E}^{\lambda}\left(\frac{x+y}{2}\right)>\frac{\mathcal{E}^{\lambda}(x)+\mathcal{E}^{\lambda}(y)}{2}
$$

for all $x, y \in(0,1)$ with $x \neq y$, which implies that the function $\mathcal{E}^{\lambda}(t)$ is strictly concave on $(0,1)$.

Note that

$$
t^{-1} \mathcal{E}^{\lambda-1}(t)[\mathcal{E}(t)-\mathcal{K}(t)]=\frac{1}{\lambda} \frac{d \mathcal{E}^{\lambda}(t)}{d t}
$$

Therefore, Lemma 2.6 follows easily from $(2.22)$ and the concavity of $\mathcal{E}^{\lambda}(t)$ on $(0,1)$.

Lemma 2.7 Let $\lambda=2 \log 2 /[2 \log \pi-\log 2-2 \log \mathcal{E}(\sqrt{2} / 2)]=1.3930 \ldots$

$$
h_{1}(t)=\frac{2^{1+\lambda}}{\pi^{\lambda}} \mathcal{E}^{\lambda}(t)-\frac{(5+t)^{\lambda}+(5-7 t)^{\lambda}}{4^{\lambda}}
$$

and

$$
h_{2}(t)=\frac{2^{1+\lambda}}{\pi^{\lambda}} \mathcal{E}^{\lambda}(t)-(\sqrt{2}-2 t)^{\lambda}-2^{\lambda / 2} .
$$

Then $h_{1}(t)>0$ for $t \in[3 / 5,17 / 25)$ and $h_{2}(t)>0$ for $t \in[17 / 25, \sqrt{2} / 2)$.

Proof Simple computations lead to

$$
\begin{aligned}
& h_{1}\left(\frac{17}{25}\right)=0.0022 \ldots>0, \quad h_{2}\left(\frac{\sqrt{2}}{2}\right)=0, \\
& h_{1}^{\prime}(t)=\frac{\lambda}{4^{\lambda}}\left[\frac{2^{3 \lambda+1}}{\pi^{\lambda}} t^{-1} \mathcal{E}^{\lambda-1}(t)(\mathcal{E}(t)-\mathcal{K}(t))+7(5-7 t)^{\lambda-1}-(5+t)^{\lambda-1}\right], \\
& h_{2}^{\prime}(t)=2 \lambda\left[\left(\frac{2}{\pi}\right)^{\lambda} t^{-1} \mathcal{E}^{\lambda-1}(t)(\mathcal{E}(t)-\mathcal{K}(t))+(\sqrt{2}-2 t)^{\lambda-1}\right], \\
& h_{1}^{\prime}\left(\frac{3}{5}\right)=-0.0471 \ldots<0, \quad h_{2}^{\prime}\left(\frac{17}{25}\right)=-0.236 \ldots<0 .
\end{aligned}
$$

From (2.24) and (2.25) together with Lemma 2.6 we clearly see that both $h_{1}^{\prime}(t)$ and $h_{2}^{\prime}(t)$ are strictly decreasing on $(0, \sqrt{2} / 2)$. Then $(2.26)$ leads to the conclusion that $h_{1}(t)$ is strictly decreasing on $[3 / 5,17 / 25]$ and $h_{2}(t)$ is strictly decreasing on $[17 / 25, \sqrt{2} / 2)$.

Therefore, Lemma 2.7 follows from (2.23) and the monotonicity of $h_{1}(t)$ on [3/5,17/25] and $h_{2}(t)$ on $[17 / 25, \sqrt{2} / 2)$. 
Lemma 2.8 (See [18], Corollary 3.2) The inequality

$$
\frac{2}{\pi} \mathcal{E}(t)<\frac{\left(1-t^{2}\right)^{5 / 8}+1}{\left(1-t^{2}\right)^{1 / 8}+1}
$$

holds for all $t \in(0,1)$.

\section{Main results}

Theorem 3.1 Let $\lambda=2 \log 2 /[2 \log \pi-\log 2-2 \log \mathcal{E}(\sqrt{2} / 2)]=1.3930 \ldots$ Then the double inequality

$$
M_{p}(a, b)<T[A(a, b), Q(a, b)]<M_{q}(a, b)
$$

holds for all $a, b>0$ with $a \neq b$ if and only if $p \leq \lambda$ and $q \geq 3 / 2$.

Proof Since the arithmetic mean $A(a, b)$, quadratic mean $Q(a, b)$, Toader mean $T(a, b)$, and $r$ th power mean $M_{r}(a, b)$ are symmetric and homogeneous of degree 1 , without loss of generality, we assume that $a>b$. Let $t=(a-b) / \sqrt{2\left(a^{2}+b^{2}\right)}$. Then $t \in(0, \sqrt{2} / 2)$ and equations (1.2)-(1.5) lead to

$$
\begin{aligned}
& M_{r}(a, b)=\frac{A(a, b)}{\sqrt{1-t^{2}}}\left[\frac{\left(\sqrt{1-t^{2}}+t\right)^{r}+\left(\sqrt{1-t^{2}}-t\right)^{r}}{2}\right]^{1 / r}, \\
& T[A(a, b), Q(a, b)]=\frac{2 A(a, b) \mathcal{E}(t)}{\pi \sqrt{1-t^{2}}} .
\end{aligned}
$$

We divide the proof into three cases.

Case $1 r \geq 3 / 2$. Then it follows from (3.1) and (3.2) together with the monotonicity of $M_{r}(a, b)$ with respect to $r$ that

$$
\begin{aligned}
& T[A(a, b), Q(a, b)]-M_{r}(a, b) \\
& \leq T[A(a, b), Q(a, b)]-M_{3 / 2}(a, b) \\
&= \frac{A(a, b)}{\sqrt{1-t^{2}}}\left[\frac{2}{\pi} \mathcal{E}(t)-\frac{\left(1-t^{2}\right)^{5 / 8}+1}{\left(1-t^{2}\right)^{1 / 8}+1}\right] \\
&+\frac{A(a, b)}{\sqrt{1-t^{2}}}\left[\frac{\left(1-t^{2}\right)^{5 / 8}+1}{\left(1-t^{2}\right)^{1 / 8}+1}-\left(\frac{\left(\sqrt{1-t^{2}}+t\right)^{3 / 2}+\left(\sqrt{1-t^{2}}-t\right)^{3 / 2}}{2}\right)^{2 / 3}\right] .
\end{aligned}
$$

Therefore,

$$
T[A(a, b), Q(a, b)]<M_{r}(a, b)
$$

for all $a, b>0$ with $a \neq b$ follows from Lemmas 2.2 and 2.8 together with (3.3). 
Case $2 r \leq \lambda$. Then equations (3.1) and (3.2) together with the monotonicity of $M_{r}(a, b)$ with respect to $r$ lead to

$$
\begin{aligned}
T & {[A(a, b), Q(a, b)]-M_{r}(a, b) } \\
& \geq T[A(a, b), Q(a, b)]-M_{\lambda}(a, b) \\
& =\frac{A(a, b)}{\sqrt{1-t^{2}}}\left[\frac{2}{\pi} \mathcal{E}(t)-\left(\frac{\left(\sqrt{1-t^{2}}+t\right)^{\lambda}+\left(\sqrt{1-t^{2}}-t\right)^{\lambda}}{2}\right)^{1 / \lambda}\right] .
\end{aligned}
$$

We divide the proof into two subcases.

Subcase $2.1 t \in(0,3 / 5)$. Let $u=t / \sqrt{1-t^{2}}$. Then $u \in(0,3 / 4)$ and (3.4) leads to

$$
\begin{aligned}
T & {[A(a, b), Q(a, b)]-M_{r}(a, b) } \\
& >A(a, b)\left[\frac{2}{\pi} \sqrt{1+u^{2}} \mathcal{E}\left(\frac{u}{\sqrt{1+u^{2}}}\right)-\left(\frac{(1+u)^{\lambda}+(1-u)^{\lambda}}{2}\right)^{1 / \lambda}\right] .
\end{aligned}
$$

Therefore,

$$
T[A(a, b), Q(a, b)]>M_{r}(a, b)
$$

for $0<|a-b| / \sqrt{2\left(a^{2}+b^{2}\right)}<3 / 5$ with $a \neq b$ follows from Lemma 2.5 and (3.5).

Subcase $2.2 t \in[3 / 5, \sqrt{2} / 2)$. Let

$$
h(t)=\frac{2^{1+\lambda}}{\pi^{\lambda}} \mathcal{E}^{\lambda}(t)-\left(\sqrt{1-t^{2}}+t\right)^{\lambda}-\left(\sqrt{1-t^{2}}-t\right)^{\lambda} .
$$

It is easy to verify that

$$
\sqrt{1-t^{2}} \leq \frac{5-3 t}{4} \text { and } \sqrt{1-t^{2}}<\sqrt{2}-t
$$

for all $t \in(0, \sqrt{2} / 2)$.

Equation (3.6) and inequality (3.7) lead to

$$
h(t)>\frac{2^{1+\lambda}}{\pi^{\lambda}} \mathcal{E}^{\lambda}(t)-\frac{(5+t)^{\lambda}+(5-7 t)^{\lambda}}{4^{\lambda}}
$$

and

$$
h(t)>\frac{2^{1+\lambda}}{\pi^{\lambda}} \mathcal{E}^{\lambda}(t)-(\sqrt{2}-2 t)^{\lambda}-2^{\lambda / 2} .
$$

Therefore,

$$
T[A(a, b), Q(a, b)]>M_{r}(a, b)
$$

for $3 / 5 \leq|a-b| / \sqrt{2\left(a^{2}+b^{2}\right)}$ with $a \neq b$ follows from Lemma 2.7, (3.4), (3.6), (3.8), and (3.9). 
Case $3 \lambda<r<3 / 2$. On the one hand, equations (1.2) and (1.5) lead to

$$
\begin{aligned}
\lim _{x \rightarrow 0^{+}} & {\left[\log T[A(1, x), Q(1, x)]-\log M_{r}(1, x)\right] } \\
& =\log \left[\frac{\sqrt{2} \mathcal{E}\left(\frac{\sqrt{2}}{2}\right)}{\pi}\right]+\frac{\log 2}{r} \\
& =-\frac{(r-\lambda) \log 2}{\lambda r}<0 .
\end{aligned}
$$

Inequality (3.10) implies that there exists $\delta_{1}>0$ such that

$$
T[A(a, b), Q(a, b)]<M_{r}(a, b)
$$

for all $a, b>0$ with $a / b \in\left(0, \delta_{1}\right)$.

On the other hand, by the Taylor expansion and let $x>0$ and $x \rightarrow 0$, then equations (1.2) and (1.5) lead to

$$
\begin{aligned}
T & {[A(1,1-x), Q(1,1-x)]-M_{r}(1,1-x) } \\
& =\frac{2}{\pi} \sqrt{1-x+\frac{x^{2}}{2}} \mathcal{E}\left(\frac{x}{2 \sqrt{1-x+\frac{x^{2}}{2}}}\right)-\left[\frac{1+(1-x)^{r}}{2}\right]^{1 / r} \\
& =1-\frac{x}{2}+\frac{x^{2}}{16}-\left[1-\frac{x}{2}+\left(\frac{1}{16}-\frac{3-2 r}{16}\right) x^{2}\right]+o\left(x^{2}\right) \\
& =\frac{3-2 r}{16} x^{2}+o\left(x^{2}\right) .
\end{aligned}
$$

Equation (3.11) implies there exists $\delta_{2} \in(0,1)$ such that

$$
T[A(a, b), Q(a, b)]>M_{r}(a, b)
$$

for all $a, b>0$ with $a / b \in\left(1-\delta_{2}, 1\right)$.

From Theorem 3.1 we get Corollary 3.2 immediately.

Corollary 3.2 Let $\lambda=2 \log 2 /[2 \log \pi-\log 2-2 \log \mathcal{E}(\sqrt{2} / 2)]=1.3930 \ldots$. Then the double inequality

$$
\frac{\pi}{2}\left[\frac{\left(\sqrt{1-t^{2}}+t\right)^{p}+\left(\sqrt{1-t^{2}}-t\right)^{p}}{2}\right]^{1 / p}<\mathcal{E}(t)<\frac{\pi}{2}\left[\frac{\left(\sqrt{1-t^{2}}+t\right)^{q}+\left(\sqrt{1-t^{2}}-t\right)^{q}}{2}\right]^{1 / q}
$$

holds for all $t \in(0, \sqrt{2} / 2)$ if and only if $p \leq \lambda$ and $q \geq 3 / 2$.

Competing interests

The authors declare that they have no competing interests.

Authors' contributions

All authors contributed equally to the writing of this paper. All authors read and approved the final manuscript.

\section{Author details}

'School of Mathematics and Computation Science, Hunan City University, Yiyang, 413000, China. ${ }^{2}$ Department of Mathematics, Hangzhou Normal University, Hangzhou, 310036, China. 


\section{Acknowledgements}

This research was supported by the Natural Science Foundation of China under Grants 11301127, 11371125, 11401191, and 61374086, the Natural Science Foundation of Zhejiang Province under Grant LY13A010004 and the Natural Science Foundation of Hunan Province under Grant 12C0577.

\section{Received: 23 September 2015 Accepted: 1 December 2015 Published online: 22 December 2015}

\section{References}

1. Toader, G: Some mean values related to the arithmetic-geometric mean. J. Math. Anal. Appl. 218(2), 358-368 (1998)

2. Bullen, PS, Mitrinović, DS, Vasić, PM: Means and Their Inequalities. Reidel, Dordrecht (1988)

3. Neuman, E: Bounds for symmetric elliptic integrals. J. Approx. Theory 122(2), 249-259 (2003)

4. Kazi, H, Neuman, E: Inequalities and bounds for elliptic integrals. J. Approx. Theory 146(2), 212-226 (2007)

5. Kazi, H, Neuman, E: Inequalities and bounds for elliptic integrals II. In: Special Functions and Orthogonal Polynomials. Contemp. Math., vol. 471, pp. 127-138. Am. Math. Soc., Providence, RI (2008)

6. Anderson, GD, Vamanamurthy, MK, Vuorinen, M: Conformal Invariants, Inequalities, and Quasiconformal Maps. Wiley, New York (1997)

7. Lin, TP: The power mean and the logarithmic mean. Am. Math. Mon. 81, 879-883 (1974)

8. Pittenger, AO: Inequalities between arithmetic and logarithmic means. Univ. Beograd. Publ. Elektrotehn. Fak. Ser. Mat. 678-715, 15-18 (1980)

9. Pittenger, AO: The symmetric, logarithmic and power means. Univ. Beograd. Publ. Elektrotehn. Fak. Ser. Mat. 678-715, 19-23 (1980)

10. Stolarsky, KB: The power and generalized logarithmic means. Am. Math. Mon. 87(7), 545-548 (1980)

11. Alzer, H: Ungleichungen für $(e / a)^{a}(b / e)^{b}$. Elem. Math. 40, 120-123 (1985)

12. Alzer, H: Ungleichungen für Mittelwerte. Arch. Math. 47(5), 422-426 (1986)

13. Burk, F: The geometric, logarithmic, and arithmetic mean inequality. Am. Math. Mon. 94(6), 527-528 (1987)

14. Alzer, H, Qiu, S-L: Inequalities for means in two variables. Arch. Math. 80(2), 201-215 (2003)

15. Costin, I, Toader, G: Optimal evaluations of some Seiffert-type means by power mean. Appl. Math. Comput. 219(9), 4745-4754 (2013)

16. Chu, Y-M, Wang, M-K, Qiu, S-L, Qiu, Y-F: Sharp generalized Seiffert mean bounds for Toader mean. Abstr. Appl. Anal. 2011, Article ID 605259 (2011)

17. Chu, Y-M, Wang, M-K: Inequalities between arithmetic-geometric, Gini, and Toader means. Abstr. Appl. Anal. 2012, Article ID 830585 (2012)

18. Chu, Y-M, Wang, M-K: Optimal Lehmer mean bounds for the Toader mean. Results Math. 61(3-4), 223-229 (2012)

19. Vuorinen, M: Hypergeometric functions in geometric function theory. In: Special Functions and Differential Equations (Madras, 1977), pp. 119-126. Allied Publ., New Delhi (1998)

20. Qiu, S-L, Shen, J-M: On two problems concerning means. J. Hongzhou Inst. Electron. Eng. 17(3), 1-7 (1997) (in Chinese)

21. Barnard, RW, Pearce, K, Richards, KC: An inequality involving the generalized hypergeometric function and the arc length of an ellipse. SIAM J. Math. Anal. 31(3), 693-699 (2000)

22. Alzer, H, Qiu, S-L: Monotonicity theorems and inequalities for the complete elliptic integrals. J. Comput. Appl. Math. 172(2), 289-312 (2004)

23. Chu, Y-M, Wang, M-K, Ma, X-Y: Sharp bounds for Toader mean in terms of contraharmonic mean with applications. J. Math. Inequal. 7(2), 161-166 (2012)

24. Hua, Y, Qi, F: A double inequality for bounding Toader mean by the centroidal mean. Proc. Indian Acad. Sci. Math. Sci. 124(4), 527-531 (2014)

25. Chu, Y-M, Wang, M-K, Qiu, S-L: Optimal combination bounds of root-square and arithmetic means for Toader mean Proc. Indian Acad. Sci. Math. Sci. 122(1), 41-51 (2012)

26. Song, Y-Q, Jiang, W-D, Chu, Y-M, Yan, D-D: Optimal bounds for Toader mean in terms of arithmetic and contraharmonic means. J. Math. Inequal. 7(4), 751-757 (2013)

27. Li, W-H, Zheng, M-M: Some inequalities for bounding Toader mean. J. Funct. Spaces Appl. 2013, Article ID 394194 (2013)

28. Hua, Y, Qi, F: The best bounds for Toader mean in terms of the centroidal and arithmetic means. Filomat 28(4), 775-780 (2014)

29. Sun, H, Chu, Y-M: Bounds for Toader mean by quadratic and harmonic means. Acta Math. Sci. 35A(1), 36-42 (2015) (in Chinese)

30. Chu, Y-M, Wang, M-K, Jiang, Y-P, Qiu, S-L: Concavity of the complete elliptic integrals of the second kind with respect to Hölder means. J. Math. Anal. Appl. 395(2), 637-642 (2012) 